\title{
Method for Optimizing the Dual of Linear Fuzzy Programming Problems
}

\author{
Frank Rogers \\ Department of Mathematics, University of West Alabama Livingston, Alabama 35470, USA \\ frogers@uwa.edu
}

\section{Abstract}

This article'sgoal is to support the existence of the dual in a Linear Fuzzy Real environment and focus on its application to linear fuzzy program problems. This concept will apply to linear fuzzy programming problems that contain fuzzy constraints with a crisp objective function, crisp constraints with a fuzzy objective function, or fuzzy constraints with a fuzzy objective function. It is also proposed here that optimizing fuzzy constraints and objectives of the dual linear program that consist of a triplet and are much like triangular fuzzy numbers, but differ in that they are a hybrid fuzzy number that contains characteristics that are both fuzzy and crisp.

\section{Mathematics Subject Classification: 90C70}

Keywords: Fuzzy linear programming; Fuzzy constraints; Linear fuzzy real number

\section{INTRODUCTION}

An idea in decision making is to maximize gains and minimize. This also applies to the world of fuzzy decision making. Fuzzy set were initially introduced by Bellman and Zadeh [1]. This concept was then adopted to mathematical programming by Tanaka et al. [9]. Fuzzy linear programming problem with fuzzy coefficients was formulated by Negoita [6]. Zimmerman [10] presented a fuzzy approach to multi-objective linear programming problems. Dubois and Prade [2] studied linear fuzzy constraints. Tankaka and Asai [8] proposed a formulation of fuzzy linear programming with fuzzy constraints and gave a method for its solution. Neggers and Kim researched fuzzy posets [4] and created Linear Fuzzy Real numbers [5]. Linear Fuzzy Real numbers were used by Monk [3] and Prevo [7] in the study of fuzzy random variables and used to optimize the primal problems of linear programs with fuzzy constraints[8]. The relationships between the primal and the dual is clear in a crisp environment. The objective of this paper is to show that the relationship is valid for an LFR environment as well.

In general, the optimal solution of the primal contains the solution of the dual. However, knowledge of the dual is important. The economic interpretation of the dual is useful in making future decisions in the activities being programmed. Also, the solution of a linear programing problem may be easier to obtain through the dual than through the primal problem.

The paper is outlined as follows. Operations on LFR are considered in Section 2. In Section 3, the study of the dual of linear programming problems with linear fuzzy real constraints. In Section 4, applications of LFR optimization are considered.

\section{Linear Fuzzy Real Numbers}

Considering the real numbers $R$, one way to associate a fuzzy number with a fuzzy subset of real numbers is as a function $\mu: R \rightarrow[0,1]$, where the value $\mu(x)$ represents a degree of belonging to the subset of R. The Linear Fuzzy Real numbers as described by Neggers and Kim [5,3] is a triple of real numbers $(a, b, c)$ where $a \leq b \leq c$ of real numbers, See Fig. 1, such that:

1. $\mu(x)=1$ if $x=b$;

2. $\mu(x)=0$ if $x \leq a$ or $x \geq c$;

3. $\mu(x)=(x-a) /(b-a)$ if $a<x<b$;

4. $\mu(x)=(c-x) /(c-b)$ if $b<x<c$.

For a real number $c$, we let $\epsilon(c)=\mu$ with associated triple (c,c,c). Then $\mu$ is a linear fuzzy real number with $\mu(c)=1$ and $\mu(x)=0$ otherwise. As a linear fuzzy real number we consider $\epsilon(c)=\mu$ to represent the real number $c$ itself. Thus by this interpretation we note that the set $R$ of all real numbers is a subset of the set containing the linear fuzzy real numbers. The set of the linear fuzzy real numbers is a hybrid set showing intermediate properties, 


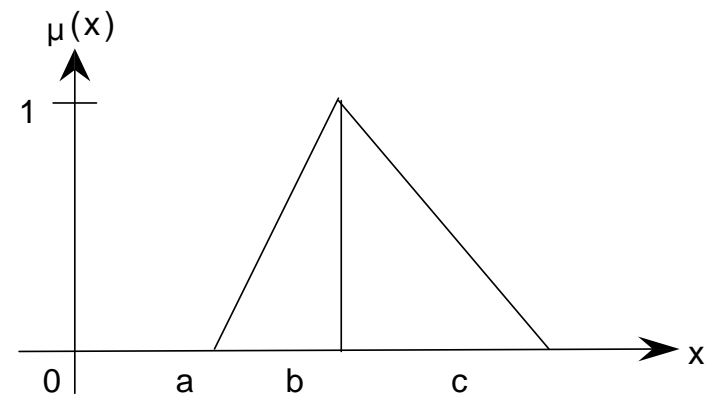

Fig. 1. Linear Fuzzy Real number $\mu(a, b, c)$

which are unique to the set and not those of either the real numbers or the "general" fuzzy numbers.

LetLFR $=\{\mu: R \rightarrow[0,1] \mid \mu$ is a linear fuzzy real number $\}$. Each $\mu$ has a set of descriptive parameters. The base is defined as the triple $(a, b, c)$ that occurs in the definition of a linear fuzzy real number. Thus one may write an element of LFR as $\mu=\mu(a, b, c)$.

\subsection{Addition and Subtraction}

Given the linear fuzzy real numbers $\mu_{1}=\mu\left(a_{1}, b_{1}, c_{1}\right)$ and $\mu_{2}=\mu\left(a_{2}, b_{2}, c_{2}\right)$,

$\mu_{1}+\mu_{2}$ is defined by

$\mu_{1}+\mu_{2}=\mu\left(a_{1}+a_{2}, b_{1}+b_{2}, c_{1}+c_{2}\right)$.

This operation is not the usual definition of addition of functions. It is also clear that $\mu+\epsilon(0)=\mu$ for all $\mu \in$ LFR. For subtraction, we have

$$
\mu_{1}-\mu_{2}=\mu\left(a_{1}-c_{2}, b_{1}-b_{2}, c_{1}-a_{2}\right) \text {. }
$$

\subsection{Law of trichotomy}

A linear fuzzy real number $\mu(a, b, c)$ is defined to be positive if $a>0$, negative if $c<0$, and zeroic if $a \leq 0$ and $c \geq 0$. The following properties also hold:

1. If $\mu$ is positive, then $-\mu$ is negative;

2. If $\mu$ is negative, then $-\mu$ is positive;

3. If $\mu$ is zeroic, then $-\mu$ is also zeroic;

4. If $\mu_{1}$ and $\mu_{2}$ are positive, then so is $\mu_{1}+\mu_{2}$;

5. If $\mu_{1}$ and $\mu_{2}$ are negative, then so is $\mu_{1}+\mu_{2}$;

6. If $\mu_{1}$ and $\mu_{2}$ are zeroic, then so is $\mu_{1}+\mu_{2}$;

7. For any $\mu, \mu-\mu$ is zeroic.

\subsection{Multiplication and Division}

Given the linear fuzzy real numbers $\mu_{1}=\mu\left(a_{1}, b_{1}, c_{1}\right)$ and $\mu_{2}=\mu\left(a_{2}, b_{2}, c_{2}\right)$,

$\mu_{1} \cdot \mu_{2}$ is defined by

$\mu_{1} \cdot \mu_{2}=\mu\left(\min \left\{a_{1} a_{2}, a_{1} c_{2}, a_{2} c_{1}, c_{1} c_{2}\right\}, b_{1} b_{2}, \max \left\{a_{1} a_{2}, a_{1} c_{2}, a_{2} c_{1}, c_{1} c_{2}\right\}\right)$.

Given the linear fuzzy real numbers $\mu_{1}=\mu\left(a_{1}, b_{1}, c_{1}\right)$ and $\mu_{2}=\mu\left(a_{2}, b_{2}, c_{2}\right), \frac{\mu_{1}}{\mu_{2}}$ is defined by

$\frac{\mu_{1}}{\mu_{2}}=\mu_{1} \cdot \frac{1}{\mu_{2}}$,

where $\frac{1}{\mu_{2}}=\mu\left(\min \left\{\frac{1}{a_{2}}, \frac{1}{b_{2}}, \frac{1}{c_{2}}\right\}\right.$, median $\left.\left\{\frac{1}{a_{2}}, \frac{1}{b_{2}}, \frac{1}{c_{2}}\right\}, \max \left\{\frac{1}{a_{2}}, \frac{1}{b_{2}}, \frac{1}{c_{2}}\right\}\right)$.

\subsection{Functions on LFR}

Given a function $\mathrm{f}: \mathrm{R} \rightarrow \mathrm{R}$ and $\mu(\mathrm{a}, \mathrm{b}, \mathrm{c}) \in \mathrm{LFR}, \mathrm{f} *(\mu): \mathrm{LFR} \rightarrow \mathrm{LFR}$ is defined as

$f *(\mu)=\mu\left(a^{*}, b^{*}, c^{*}\right)$,

where $a^{*}=\min \{f(a), f(b), f(c)\}, b^{*}=\operatorname{median}\{f(a), f(b), f(c)\}, c^{*}=\max \{f(a)$, 
$f(b), f(c)\}$. If $a=b$ or $b=c$, then $a^{*}=b *$ or $b^{*}=c^{*}$. Therefore if $a=b=c$ then it follows that $a^{*}=b^{*}=c^{*}$, i.e., $f^{*}(\epsilon(b))=$ $\epsilon(f(b))$. Hence $f *$ is an extension of the function $f$.

\subsection{Linear equations on LFR}

Before discussing linear programming in full detail, we must discuss linear equations in the LFR system. A linear equation over LFR is an equation of the form

$\mu_{1} \cdot \mu_{x}+\mu_{2}=\mu_{3} \cdot \mu_{x}+\mu_{4}$

where the $\mu_{i}$ are LFR's for $i=1,2,3,4$ and $\mu_{x}$ is an unknown LFR with a triple of unknown real numbers $(\alpha, \beta, \gamma)$. The solution set of the general linear equation can be roughly classified as

1. empty set,

2. singleton set,

3. not a singleton set but a bounded set:

$\beta_{1} \leq \alpha \leq \beta \leq \gamma \leq \beta_{2}$ for $\beta_{1}, \beta_{2} \in R$,

4. an unbounded set but not all LFR's are included,

5. all possible LFR's are included.

An acceptable solution, given the choices of the solution set, depends on the parameters of the linear program. For the purpose of solving fuzzy linear programs, classification 2, 3, and 5 are acceptable. A solution set that is bounded but not a singleton would imply that $\mu_{\mathrm{x}}$ is not equal to the solution set in a crisp sense. Solving these equations through traditional means can be a daunting task. If we define a relation $\mu_{1} \equiv \mu_{2}(\bmod \theta)$ iff $\mu_{1}-\mu_{2}$ is zeroic, then $\mu(a, b, c) \equiv$ $\epsilon(b)(\bmod \theta)$ since $\mu(a, b, c)-\epsilon(b)=\mu(a-b, 0, c-b)$. Therefore if we define $\left[\left[\mu_{1}\right]=\left\{\mu_{2} \mid \mu_{2} \equiv \mu_{1}(\bmod \theta)\right.\right.$, then $[\mu(a, b, c)]=[\epsilon(b)]$. Furthermore, in order that $\epsilon(a) \equiv \epsilon(b)(\bmod \theta)$, we must have $\epsilon(a)-\epsilon(b)=\epsilon(a-b)$ zeroic, which can only happen if $a=b$. Hence, we have a mapping $\Phi: \mu \rightarrow[\mu]$ with the property that if we compose this with the mappingb $\rightarrow \epsilon($ b) then we obtain the sequence $R \stackrel{\epsilon}{\rightarrow}$ LFR $\stackrel{\varphi}{\rightarrow} L F R / Z$, where $Z$ is the set of zeroic elements of LFR, whence $L F R / Z$ is seen to be isomorphic to itself. If $: Z \rightarrow L F R$ is the inclusion mapping, then we obtain a further diagram:

$$
\mathrm{Z} \stackrel{\sigma}{\rightarrow} \mathrm{LFR} \stackrel{\varphi}{\rightarrow} \mathrm{LFR} / \mathrm{Z} \stackrel{\epsilon}{\rightarrow} \mathrm{LFR} .
$$

$\operatorname{Thus}\left[\mu * \mu^{-1}\right]=[\mu] *\left[\mu^{-1}\right]=\epsilon(1)$, i.e., $[\mu]$ has a multiplicative inverse in LFR/Z. The properties of LFR/Z allow one to solve for the solution of fuzzy linear equations using the inverse order of operations.

\section{$3 \quad$ Linear fuzzy programming}

Linear programming models are a special kind of decision model: The decision space is defined by constraints and an objective function. Thus decisionmaking occurs under certainty. In linear fuzzy programming, optimization occurs under uncertainty. For linear fuzzy programming we will need to depart from the classical assumption of linear programming. The classical assumption is that our objective and constraints are "crisp". In addition, the term "to maximize" or "to minimize" is used in its strictest sense. Despite the unique properties of the LFR system, it is nevertheless a fuzzy environment.

Thus we can use the nature of LFR to solve linear programming problems in a fuzzy environment. This offers many possibilities for the decision maker. First he may not want to actually maximize or minimize the objective function. He may want to reach a decision that is not crisply definable as optimal. The actual purpose may be to improve the profit situation for example.

Secondly, the constraints might be vague. The s sign might not be defined in the traditional sense to the degree that smaller violations may be acceptable. This can happen when the constraints represent aspiration levels that are not definable crisply.

Finally, the role of the constraints can be different from the ones in the classical sense in that the violation of any single constraint may not render the solution infeasible. The decision maker might accept small violations of constraint but might define different degrees of importance to violations of different constraints.

\subsection{Maximal/Minimal element}

If $U \subseteq L F R$, then it is a partially ordered set. An element $\mu_{\min } \in U$ is called a minimal element of $U$ if no element is less than $\mu_{\min }$. Similarly an element $\mu_{\max } \in \mathrm{U}$ is called a maximal element of LFR if no element is greater than $\mu_{\max }$. Note that since the LFR's are not linearly ordered it can have more than one minimal or maximal element. The actual search for this element requires a series of calculations. However, before further discussing this we will need the following definitions and propositions.

Definition 3.1. A set $V$ in $R^{n}$ is said to be a linear variety if given any $x, y \in V$ we have $\lambda x+(1-\lambda) y \in V$ for all real numbers $\lambda$. 
Definition 3.2. A hyperplane in $\mathrm{R}^{\mathrm{n}}$ is an $(\mathrm{n}-1)$ dimensional linear variety.

Proposition 3.3. Let $H=\left\{\mu_{x} \in[L F R / Z]^{n}: p^{\top} \mu_{x}=q\right\}$. Then $H$ is a linear variety.

Proof. Suppose $\mu_{x 1}, \mu_{x 2} \in H$, then $p^{\top}\left(\lambda \mu_{x 1}+(1-\lambda) \mu_{x 2}\right)=\lambda p^{\top} \mu_{x 1}+p^{\top} \mu_{x 2}-\lambda p^{\top} \mu_{x 2}=\lambda q+q-\lambda q=q$. Therefore $H$ is a linear variety.

Proposition 3.4. Let $H=\left\{\mu_{x} \in[L F R / Z]^{n}: p^{\top} \mu_{x}=q\right\}$. Then $H$ is a hyperplane.

Proof. It follows from the previous proposition that $\mathrm{H}$ is a linear variety. Let $\mu_{x 1}$ be any fuzzy vector in $\mathrm{H}$. We can obtain the set $G=H-\mu_{x 1}$ which is a linear subspace of $[L F R / Z]^{n}$. This subspace consists of all vectors $\mu_{x}$ satisfying $p^{\top} \mu_{x}=0$; thus the vectors $\mu_{x}$ are orthogonal to $p$. Therefore $H$ is an $(n-1)$ dimensional subspace (hyperplane).

We have observed above that "up to Zeroic" the solution of linear equations depends on the solution of crisp equations involving the middle values, i.e., the b's in $\mu(a, b, c)$. The subsequent analysis depends on dealing with the inequalities of the type $a \leq b \leq c$. The same principle underlies our present observations.

The propositions give us an idea of the geometry of a fuzzy linear program in this setting. Hence, they play an important role in searching for the fuzzy maximal/minimal point.

\subsection{Extreme points}

Because of the previous findings we know that a fuzzy linear program problem consists of fuzzy constraints of equality and inequality. The "Modulo Z" graph of each constraint defined by an inequality is a closed half space. Similarly, the graph of each fuzzy constraint defined by equality is a hyperplane or intersection of two closed half spaces. Hence, also in this manner the set of points that satisfy the constraints of the linear programming problem is the intersection of the closed half spaces determined by the constraints. We can find the optimal solution by evaluating the basic feasible solutions. These basic feasible solutions are the extreme points. The nature of this fuzzy algorithm developed for this problem is essentially an analog of its crisp counterpart with modifications naturally.

\subsection{Fuzzy algorithm}

A fuzzy algorithm seeks to solve a problem through a series of logical operations. With a fuzzy algorithm we do not seek precision answers. Problems of the precision class are naturally restrictive and thus there arises a need for fuzzy algorithms applicable to fuzzy situations. The following algorithm evaluates each of the extreme points in LFR/Z and stores its LFR counterpart. Thus our optimal solution has a fuzzy and a crisp version.

Schematic representation of the simplex algorithm may be summarized in the following:

- First Phase: Formulate the problem and the objective function.

- Second Phase: Design an initial program.

- Third Phase: Test the given program for optimality.

- If optimal, the problem is solved.

- If not, revise the program.

We modify the simplex algorithm to LFR in the fuzzy algorithm in the following 4 steps.

\subsubsection{Step 1: Formulate the problem and the objection function}

(a) Translate the technical specification of the problem into a fuzzy inequalityand make a statement. Thus we should have a fuzzy objective and constraints, for example

Minimize $\mathrm{p}^{\top} \mu_{\mathrm{x}}$

subject to:

$M \mu_{x} \leq \mu_{b}$

$\mu_{x} \geq \epsilon(0)$.

(b) Convert the fuzzy inequality into a fuzzy equality by the addition of nonnegative slack variables.

(c) Modify the objective function to include the slack variables.

(d) Modify the main body to consist of a "tri-matrix", such that one matrixof triplets becomes three matrices of singlets. Hence we let $\left(\mu_{\mathrm{ij}}\right)=(\mathrm{A}, \mathrm{B}, \mathrm{C})$, where $\mathrm{A}=\left(\mathrm{a}_{\mathrm{ij}}\right), \mathrm{B}=\left(\mathrm{b}_{\mathrm{ij}}\right)$, and $\mathrm{C}=\left(\mathrm{c}_{\mathrm{ij}}\right)$.

\subsubsection{Step 2: Design an initial program (a basic feasible solution)}

Design the first program so that only the slack variables are included in the solution. Place this program in a simplex tableau. In the objective row, above each column variable, place the corresponding coefficient of that variable from Step 1(c). 


\subsubsection{Step 3: Test and revise the program}

(a) Use the matrix $B=\left(b_{i j}\right)$ to calculate the net evaluation row. To get a number in the net evaluation row under a column, multiply the entries in that column by the corresponding numbers in the objective column, and add the products. Then subtract this sum from the number listed in the objective row at the top of the column. Enter the net evaluation row under the column.

(b) Test the entries in the net evaluation row for the given simplex tableau.If all the entries are zero or positive, the optimal solution has been obtained. Otherwise, the presence of any positive entry in the evaluation row indicates that a better program can be obtained.

(c) Revise the program:

(c.1) Find the key column, which consists of the column under which fallsthe largest negative netevaluation. Find the key row using the key number. Divide the entries in the "quantity" column by the corresponding nonnegative entries of the key column to form replacement ratios, and compare these ratios. The row that contains the smallest replacement ratio is the key row and the key column is the key number.

(c.2) Transform the key row. Divide all the numbers in the key row and thecorresponding row for $\mathrm{A}$ and $\mathrm{C}$.

(c.3) Transform the non-key rows. Subtract from the old row number of agiven non-key row (in each column) the product of the corresponding keyrow number and the corresponding fixed ratio formed by dividing the old row number in the key column by the key number.

(c.4) Perform identical arithmetic operations on A and C. (Note that in key row the values of A and C will switch due to the nature of LFR.) The result will give the corresponding new row number. Make this transformation for all the non-key rows.

(c.5) Enter the result of Step 3 above in a tableau representing the revisedprogram.

\subsubsection{Step 4:}

Obtain the optimal program by repeating Step 3 until an optimal program has been derived.

\subsection{Support of the dual in LFR}

The application of LFR to the primal problem was shown in my previous article. Here we shall discuss the dual problem. The dual problem shall be defined as follows:

Given that the primal problem:

Minimize $c^{\top} x$

Such that

$A \mu_{x \leqq} \mu_{b}$

$\mu_{\mathrm{x}} \geq \varepsilon(0)$

Then the dual problem is:

Maximize $b^{\top} w$

Such that

$A \mu_{w} \mu_{c}$

$\mu_{w} \geq \varepsilon(0)$

For dual and primal problems in LFR the following is true.

1. Given a primal problem the dual of its dual problem is again the primal problem.

2. If either the primal or dual problem has a feasible solution with a finite optimal objective value, then the other problem has a feasible solution with the same objective value.

3. If $\mu_{\mathrm{x} 0}$ is a feasible solution to the primal problem and if $\mu_{\mathrm{w}_{0}}$ is a feasible solution to the dual problem then the primal and dual problem have an optimal solution and $c^{\top} x_{0}=b^{\top} w_{0}$. To prove this we must introduce the following proposition:

\section{Proposition 3.5:}

Let $\mathrm{C}$ be a convex set such that $\mathrm{C} \subseteq(\mathrm{LFR} / \mathrm{Z})^{\mathrm{n}} \subseteq(L F R)^{n}$. Also, let $\mu_{\mathrm{y}}$ be an LFR point exterior to the closure of $\mathrm{C}$. Then for some vector $v, v^{\top} \mu_{y}<\operatorname{infv}^{\top} \mu_{x}$.

\section{Proof:}

Let $S$ be a closed ball centered at $\mu_{y}$ with radius large enough such that $S$ intersects $X$. Then the intersection formed by $S$ and $X$ is compact. If we let 
$\partial=\inf \left|\mu_{x}-\mu_{y}\right|>0$, there exist a $\mu_{x 0}$ such that $\partial=\left|\mu_{x 0}-\mu_{y}\right|$. This follows since

$f\left(\mu_{x}\right)=\left|\mu_{x}-\mu_{y}\right|$ assumes it minimum value there.

If $\mu_{x} \in X$ and $0<\alpha<1$, then $\mu_{y}=\alpha \mu_{x}+(1-\alpha) \mu_{x 0} \in \hat{C}$.

Thus

$\left|\mu_{x 0}-\mu_{y}\right|^{2} \leq\left|\alpha \mu_{x}+(1-\alpha) \mu_{x 0}-\mu_{y}\right|^{2}=\left|\mu_{x 0}-\mu_{y}+\alpha\left(\mu_{x}-\mu_{x 0}\right)\right|^{2}=$

$\left|\mu_{x 0}-\mu_{y}\right|^{2}+2 \alpha\left(\mu_{x}-\mu_{x 0}\right)\left(\mu_{x 0}-\mu_{y}\right)^{\top}+\alpha^{2}\left|\mu_{x}-\mu_{x 0}\right|^{2}$

Therefore

$0 \leq 2 \alpha\left(\mu_{x}-\mu_{x 0}\right)\left(\mu_{x 0}-\mu_{y}\right)^{\top}+\alpha^{2}\left|\mu_{x}-\mu_{x 0}\right|^{2}$

$0 \leq\left(\mu_{x}-\mu_{x 0}\right)\left(\mu_{x 0}-\mu_{y}\right)^{\top}+(\alpha / 2)\left|\mu_{x}-\mu_{x 0}\right|^{2}$

If we take the limit as $\alpha \rightarrow 0$ we obtain

$0 \leq\left(\mu_{x}-\mu_{x 0}\right)\left(\mu_{x 0}-\mu_{y}\right)^{\top}$

$0 \leq\left(\mu_{x 0}-\mu_{y}\right)^{\top} \mu_{x}-\left(\mu_{x 0}-\mu_{y}\right)^{\top} \mu_{x 0}$

$\left(\mu_{x 0}-\mu_{y}\right)^{\top} \mu_{x} \geq\left(\mu_{x 0}-\mu_{y}\right)^{\top} \mu_{x 0}$

$$
\begin{aligned}
& =\left(\mu_{x 0}-\mu_{y}\right)^{\top}\left(\mu_{y}+\mu_{x 0}-\mu_{y}\right) \\
& =\left(\mu_{x 0}-\mu_{y}\right)^{\top} \mu_{x}+\left(\mu_{x 0}-\mu_{y}\right)^{\top}\left(\mu_{x 0}-\mu_{y}\right) \\
& =\left(\mu_{x 0}-\mu_{y}\right)^{\top} \mu_{x}+\partial^{2}
\end{aligned}
$$

Let us set $v=\left(\mu_{x 0}-\mu_{y}\right)$

Then $v^{\top} \mu_{y}<\operatorname{infv}{ }^{\top} \mu_{x}$

\section{Proposition 3.6:}

LFR Programming and the dual Problem

If $\mu_{x}$ is a feasible point for a linear programming problem and $\mu_{y}$ is a feasible point for the dual problem then $c^{\top} \mu_{x} \leq \mu_{y}^{\top} A \mu_{x}$ $\leq \mathrm{b}^{\top} \mu_{\mathrm{y}}$.

Proof:

Since $\mu_{x}$ and $\mu_{y}$ satisfies

$\mu_{x} \geq 0, A \mu_{x} \leq b, \mu_{y} \geq 0,-A^{\top} \mu_{y} \leq-c$

thusc ${ }^{\top} \mu_{x} \leq\left(A^{\top} \mu_{y}\right)^{\top} \mu_{x}=\mu_{y}{ }^{\top} A \mu_{x} \leq \mu_{y}{ }^{\top} * b=b^{\top} \mu_{y}$.

With proposition 3.5 and 3.6 in place we now introduce the following proposition.

\section{Proposition 3.7:}

If the primal and dual problem have feasible solutions then the primal problem has an optimal solution $\mu_{x 0}$, and the dual problem has an optimal solution $\mu_{\mathrm{w} 0}$, and

$c^{\top} \mu_{x 0}=b^{\top} \mu_{w 0}$.

\section{Proof:}

Let $\mu_{x}$ be a feasible solution to the primal and $\mu_{w}$ be a feasible solution to the dual. By Proposition $3.6 c^{\top} \mu_{x} \leq b^{\top} \mu_{w}$. Since the object function of the primal problem is bounded and the set of feasible solutions is not empty, there is a finite optimal solution $\mu_{\mathrm{x} 0}$. By Proposition 3.5 there exists a feasible solution $\mu_{\mathrm{w} 0}$ such that $c^{\top} \mu_{\mathrm{x} 0}=\mathrm{b}^{\top} \mu_{\mathrm{w} 0}$. Now suppose $\mu_{\mathrm{w} 1}$ is any feasible solution to the dual problem, then by theorem $A$

$b^{\top} \mu_{w 0}=c^{\top} \mu_{x 0} \leq b^{\top} \mu_{w 1}$, and thus $\mu_{w 0}$ is an optimal solution to the dual.

\subsection{Example of the dual linear fuzzy programming problem}

Given the primal problem

Maximize

$$
\mu(1.75,4,5.25) \mu_{\mathrm{x} 1}+\mu(1,3,4) \mu_{\mathrm{x} 2}+\mu(2.5,3,5) \mu_{\mathrm{x} 3}
$$

Subject to

$$
\begin{gathered}
\mu(0.5,1,1.5) \mu_{\mathrm{x} 1}+\mu(1,2,2) \mu_{\mathrm{x} 2} \leq-2 \\
3 \mu_{\mathrm{x} 1}+\mu_{\mathrm{x} 2}+\mu_{\mathrm{x} 3} \leq-4
\end{gathered}
$$




$$
\begin{aligned}
& 4 \mu_{\mathrm{x} 3} \leq-1 \\
& \mu(0.5,1,1.5) \mu_{\mathrm{x} 1}+\mu_{\mathrm{x} 3} \leq-1
\end{aligned}
$$

Then the dual with slack variables yield.

Minimize $-2 \mu_{\mathrm{y} 1}-4 \mu_{\mathrm{y} 2}-\mu_{\mathrm{y} 3}-\mu_{\mathrm{y} 4}$

Subject to :

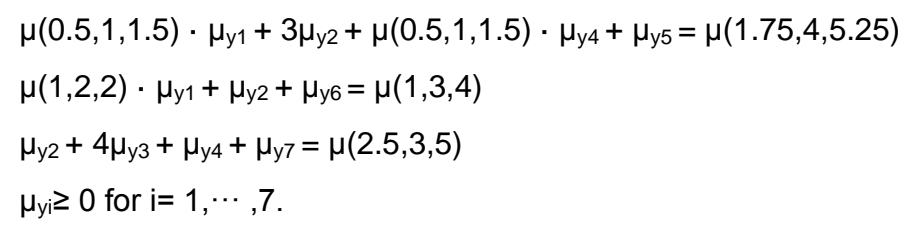

Using the Fuzzy algorithm given above, we solve this minimization problem in the following Tables 1 through 4. Each table shows a "tri-matrix" of tableaus.

\begin{tabular}{|c|c|c|c|c|c|c|c|c|c|}
\hline Tableau & basic & $\mu \mathrm{y} 1$ & ну2 & $\mu \mathrm{y} 3$ & $\mu \mathrm{y} 4$ & $\mu \mathrm{y} 5$ & $\mu y 6$ & $\mu y 7$ & $\mu_{b}$ \\
\hline \multirow[t]{4}{*}{$A$} & $(-z)$ & -2 & -4 & -1 & -1 & 0 & 0 & 0 & 0 \\
\hline & нy5 & 0.5 & 3 & 0 & 0.5 & 1 & 0 & 0 & 1.75 \\
\hline & $\mu y 6$ & 1 & 1 & 0 & 0 & 0 & 1 & 0 & 1 \\
\hline & $\mu y 7$ & 0 & 1 & 4 & 1 & 0 & 0 & 1 & 2.5 \\
\hline \multirow[t]{4}{*}{$B$} & $(-z)$ & -2 & -4 & -1 & -1 & 0 & 0 & 0 & 0 \\
\hline & нy5 & 1 & $3 * *$ & 0 & 1 & 1 & 0 & 0 & 4 \\
\hline & $\mu \mathrm{y} 6$ & 2 & 1 & 0 & 0 & 0 & 1 & 0 & 3 \\
\hline & $\mu y 7$ & 0 & 1 & 4 & 1 & 0 & 0 & 1 & 3 \\
\hline \multirow[t]{4}{*}{$\mathrm{C}$} & $(-z)$ & -2 & -4 & -1 & -1 & 0 & 0 & 0 & 0 \\
\hline & нy5 & 1.5 & 3 & 0 & 1.5 & 1 & 0 & 0 & 5.25 \\
\hline & нy6 & 2 & 1 & 0 & 0 & 0 & 1 & 0 & 4 \\
\hline & $\mu \mathrm{y} 7$ & 0 & 1 & 4 & 1 & 0 & 0 & 1 & 5 \\
\hline
\end{tabular}

Table 1. First set of tableaus

(** indicates the pivot element in Table 1 through Table 8.) 
Table 2. Second set of tableaus

\begin{tabular}{|c|c|c|c|c|c|c|c|c|c|}
\hline Tableau & basic & $\mu \mathrm{y} 1$ & $\mu \mathrm{y} 2$ & ну3 & $\mu \mathrm{y} 4$ & & $\mu y 6$ & $\mu y 7$ & $\mu_{b}$ \\
\hline \multirow[t]{4}{*}{ A } & $(-z)$ & -1.33 & 0 & -1 & -0.33 & 1.33 & 0 & 0 & 2.33 \\
\hline & нy2 & 0.17 & 1 & 0 & 0.17 & 0.33 & 0 & 0 & 0.58 \\
\hline & $\mu \mathrm{y} 6$ & 0.5 & 0 & 0 & -0.5 & -0.33 & 1 & 0 & -0.75 \\
\hline & $\mu y 7$ & -0.5 & 0 & 4 & 0.5 & -0.33 & 0 & 1 & 0.75 \\
\hline \multirow[t]{4}{*}{$B$} & $(-z)$ & -0.67 & 0 & -1 & 0.33 & 1.33 & 0 & 0 & 5.33 \\
\hline & нy2 & 0.33 & 1 & 0 & 0.33 & 0.33 & 0 & 0 & 1.33 \\
\hline & $\mu \mathrm{y} 6$ & 1.67 & 0 & 0 & -0.33 & -0.33 & 1 & 0 & 1.67 \\
\hline & $\mu \mathrm{y} 7$ & $\begin{array}{l}-0.33 \\
\end{array}$ & 0 & $4 * *$ & 0.67 & -0.33 & 0 & 1 & 1.67 \\
\hline \multirow[t]{4}{*}{$\mathrm{C}$} & $(-z)$ & 0 & 0 & -1 & 1 & 1.33 & 0 & 0 & 7 \\
\hline & нy2 & 0.5 & 1 & 0 & 0.5 & 0.33 & 0 & 0 & 1.75 \\
\hline & $\mu \mathrm{y} 6$ & 1.83 & 0 & 0 & -0.17 & -0.33 & 1 & 0 & 3.42 \\
\hline & $\mu y 7$ & -0.17 & 0 & 4 & 0.83 & -0.33 & 0 & 1 & 4.42 \\
\hline
\end{tabular}

Table 3. Third set of tableaus

\begin{tabular}{|l|l|l|l|l|l|l|l|l|l|}
\hline Tableau & basic & $\mu \mathrm{y} 1$ & $\mu \mathrm{y} 2$ & $\mu \mathrm{y} 3$ & $\mu \mathrm{y} 4$ & $\mu \mathrm{y} 5$ & $\mu \mathrm{y} 6$ & $\mu \mathrm{y} 7$ & $\mu_{\mathrm{b}}$ \\
\hline \multirow{4}{*}{$\mathrm{A}$} & $(-\mathrm{z})$ & -1.46 & 0 & 0 & -0.21 & 1.25 & 0 & 0.25 & 2.52 \\
\cline { 2 - 10 } & $\mu \mathrm{y} 2$ & 0.17 & 1 & 0 & 0.17 & 0.33 & 0 & 0 & 0.58 \\
\cline { 2 - 10 } & $\mu \mathrm{y} 6$ & 0.5 & 0 & 0 & -0.5 & -0.33 & 1 & 0 & -0.75 \\
\cline { 2 - 10 } & $\mu \mathrm{y} 3$ & -0.13 & 0 & 1 & 0.13 & -0.08 & 0 & 0.25 & 0.19 \\
\hline \multirow{4}{*}{$\mathrm{B}$} & $(-\mathrm{z})$ & -0.75 & 0 & 0 & 0.5 & 1.25 & 0 & 0.25 & 5.75 \\
\cline { 2 - 10 } & $\mu \mathrm{y} 2$ & 0.33 & 1 & 0 & 0.33 & 0.33 & 0 & 0 & 1.33 \\
\cline { 2 - 10 } & $\mu \mathrm{y} 6$ & $1.67 * *$ & 0 & 0 & -0.33 & -0.33 & 1 & 0 & 1.67 \\
\cline { 2 - 10 } & $\mu \mathrm{y} 3$ & -0.08 & 0 & 1 & 0.17 & -0.08 & 0 & 0.25 & 0.42 \\
\hline \multirow{5}{*}{$\mathrm{C}$} & $(-\mathrm{z})$ & -0.04 & 0 & 0 & 1.21 & 1.25 & 0 & 0.25 & 8.1 \\
\cline { 2 - 10 } & $\mu \mathrm{y} 2$ & 0.5 & 1 & 0 & 0.5 & 0.33 & 0 & 0 & 1.75 \\
\cline { 2 - 10 } & $\mu \mathrm{y} 6$ & 1.83 & 0 & 0 & -0.17 & -0.33 & 1 & 0 & 3.42 \\
\cline { 2 - 9 } & $\mu \mathrm{y} 3$ & -0.04 & 0 & 1 & 0.21 & -0.08 & 0 & 0.25 & 1.1 \\
\hline
\end{tabular}


Table 4. Fourth set of tableaus

\begin{tabular}{|l|l|l|l|l|l|l|l|l|l|}
\hline Tableau & basic & $\mu \mathrm{y} 1$ & $\mu \mathrm{y} 2$ & $\mu \mathrm{y} 3$ & $\mu \mathrm{y} 4$ & $\mu \mathrm{y} 5$ & $\mu \mathrm{y} 6$ & $\mu \mathrm{y} 7$ & $\mu_{\mathrm{b}}$ \\
\hline \multirow{4}{*}{$\mathrm{A}$} & $(-\mathrm{z})$ & -1.23 & 0 & 0 & -0.43 & 1.1 & 0.45 & 0.25 & 2.18 \\
\cline { 2 - 10 } & $\mu \mathrm{y} 2$ & -0.2 & 1 & 0 & 0.2 & 0.4 & -0.2 & 0 & -0.1 \\
\cline { 2 - 10 } & $\mu \mathrm{y} 1$ & 0.3 & 0 & 0 & -0.3 & -0.2 & 0.6 & 0 & -0.45 \\
\cline { 2 - 9 } & $\mu \mathrm{y} 3$ & -0.1 & 0 & 1 & 0.1 & -0.1 & 0.05 & 0.25 & 0.15 \\
\hline \multirow{5}{*}{$\mathrm{B}$} & $(-\mathrm{z})$ & 0 & 0 & 0 & 0.35 & 1.1 & 0.45 & 0.25 & 6.5 \\
\cline { 2 - 10 } & $\mu \mathrm{y} 2$ & 0 & 1 & 0 & 0.4 & 0.4 & -0.2 & 0 & 1 \\
\cline { 2 - 9 } & $\mu \mathrm{y} 1$ & 1 & 0 & 0 & -0.2 & -0.2 & 0.6 & 0 & 1 \\
\cline { 2 - 9 } & $\mu \mathrm{y} 3$ & 0 & 0 & 1 & 0.15 & -0.1 & 0.05 & 0.25 & 0.5 \\
\hline C & $(-\mathrm{z})$ & 0.78 & 0 & 0 & 1.13 & 1.1 & 0.45 & 0.25 & 9.64 \\
\cline { 2 - 9 } & $\mu \mathrm{y} 2$ & 0.4 & 1 & 0 & 0.6 & 0.4 & -0.2 & 0 & 1.9 \\
\cline { 2 - 9 } & $\mu \mathrm{y} 1$ & 1.1 & 0 & 0 & -0.1 & -0.2 & 0.6 & 0 & 2.05 \\
\cline { 2 - 9 } & $\mu \mathrm{y} 3$ & 0.05 & 0 & 1 & 0.2 & -0.1 & 0.05 & 0.25 & 1.28 \\
\hline
\end{tabular}

Since the objective function row has nonnegative values in B tableau in the fourth set, this set is optimal. In LFR the optimal values are

$$
\mu_{\mathrm{y} 1}=\mu(-0.45,1,2.05) ; \mu_{\mathrm{y} 2}=\mu(-0.1,1,1.9) ; \mu_{\mathrm{y} 3}=\mu(0.15,0.5,1.28) \text {. }
$$

In LFR/Z the optimal values are

$$
\mu_{\mathrm{y} 1}=\epsilon(1) ; \mu_{\mathrm{y} 2}=\epsilon(1) ; \mu_{\mathrm{y} 3}=\epsilon(0.5) .
$$

Thus the minimum value of the objective function is $\mu(2.18,6.5,9.64)$ in

LFR and our "crisp" value is $\epsilon(6.5)$ in $L F R / Z$.

\section{Conclusion}

From our example, it is clear that we can find a crisp optimum by projecting to the middle, $\mu(a, b, c) \rightarrow \epsilon(b)$. At the same time the method outlined produces a fuzzy solution in the form of an LFR expression, which can be used directly as a fuzzy value. In real world problems this may prove advantageous, particularly in situations where it is already known the "crisp optima" in the purest sense do not exist, but where $\mu(a, b, c) \rightarrow \epsilon(b)$ produces a "crisp good choice" for an optimum. Having done what has been done for linear optimization [8], in the future, LFR may be applied to not just single objective programming but multi-objective, multi-level, dynamic programming and goal programming in a Linear Fuzzy Real environment. This of course is from a theoretical viewpoint, useful lines of advance may occur in the different applied fields such as supply-allocation problems, production planning, scheduling, inventory networks, queuing systems, risk analysis, quality control, vehicle routing, finance, energy systems, capital budgeting, system reliability, manufacturing system, and pattern recognition. 


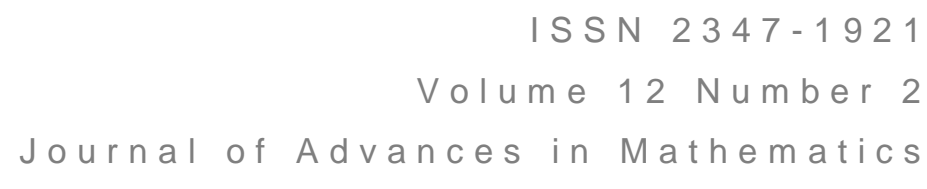

\section{References}

[1] R.E. Bellman, L.A. Zadeh, Decision making in a fuzzy environment, Management Science,17(1970), B141B164.

[2] D. Dubois, H. Prade, System of linear fuzzy constraints, Fuzzy Sets and Systems, 13 (1982), 1-10.

[3] B. Monk, A Proposed Theory of Fuzzy Random Variables, Dissertation, University of Alabama, 2001.

[4] J. Neggers, H. Kim, Fuzzy posets on sets, Fuzzy sets and systems, 17 (2001), 391-402.

[5] J. Neggers, H. Kim, On Linear Fuzzy Real Numbers, Manuscript for book under development, 2007.

[6] C.V. Negoita, Fuzziness in management, OPSA/TIMS, Miami, 1970.

[7] R. Prevo, Entropies of families of fuzzy random variables: an introduction to an in-depth exploration of several classes of important examples, Dissertation, University of Alabama, 2002.

[8] F. Rogers, J. Neggers, Y. Jun. Method for Optimizing Linear Problems with Fuzzy Constraints. International Mathematical Forum, 3 (2008), 1141-1155.

[9] H. Tanaka, K. Asai, Fuzzy linear programming problems with fuzzy numbers, Fuzzy sets and Systems, 13 (1984), 1-10.

[10] H. Tanaka, T. Okuda and K. Asai, On fuzzy mathematical programming, J. Cybernetics, 3 (1984), 37-46.

[11] H.J. Zimmermann, Fuzzy mathematical programming, Comput. Ops. Res., 10 (1983), 291-298. 